\title{
Effects of early dark energy on strong cluster lensing
}

\author{
C. Fedeli and M. Bartelmann
}

\author{
Zentrum für Astronomie, ITA, Universität Heidelberg, Albert-Überle-Str. 2, 69120 Heidelberg, Germany \\ e-mail:cosimo@ita.uni-heidelberg.de
}

Received 5 July 2006 / Accepted 5 September 2006

\begin{abstract}
We use the semi-analytic method developed earlier for computing strong-lensing optical depths to study the statistics of gravitational arcs in four dark-energy cosmologies. Specifically, we focus on models with early dark energy and compare them to more conventional models. Merger trees are constructed for the cluster population because strong cluster lensing is amplified by factors of two to three during mergers. We find that the optical depth for gravitational arcs in the early dark-energy models is increased by up to a factor of $\sim 3$ compared to the other models because of the modified dynamics of cluster formation. In particular, the probability of gravitational arcs in high-redshift clusters is considerably increased, which may offer an explanation for the unexpectedly high lensing efficiency of distant clusters.
\end{abstract}

Key words. gravitational lensing - galaxies: clusters: general - dark matter

\section{Introduction}

While the present dominance of dark energy is well-established (Goldstein et al. 2003; Hawkins et al. 2003; Spergel et al. 2003, 2006; Rebolo et al. 2004; Readhead et al. 2004; Riess et al. 2004; Tegmark et al. 2004), its evolution is largely unconstrained, in particular in the early universe. An interesting class of models for dynamical dark energy is characterised by a low but nonvanishing density for dark energy at early times (Ferreira \& Joyce 1998; Doran et al. 2001a,b; Caldwell et al. 2003; Wetterich 2004). Non-linear structure formation in this particular class of models was studied recently by Bartelmann et al. (2005) in the framework of the spherical collapse model. Interestingly, it was found that non-linear structures are expected to form substantially earlier in such early dark-energy models, if they are normalised so as to be compatible with the large-scale temperaturefluctuation amplitude of the cosmic microwave background. For two specific models with early dark energy, the population of galaxy clusters is expected to evolve by approximately one order of magnitude less strongly than in the standard $\Lambda$ CDM model.

Should this come close to reality, a rich population of massive galaxy clusters would be present at high redshift, which is completely unexpected in $\Lambda \mathrm{CDM}$. Similarly, the dynamical activity within the cluster population due to substantial mergers with sub-halos would be shifted or extended towards higher redshift.

The problem of the non-linear evolution of cosmic structures in the presence of dark energy has recently been addressed by several authors and also from a more general point of view. For example, Mota \& van de Bruck (2004), Zeng \& Gao (2005), Maor \& Lahav (2005), and Wang (2006) analyse different aspects of this issue for both constant and time-dependent equation of state parameters for the dark energy, allowing for dark energy clustering and coupling to dark matter. They outline very different properties of the final virialised objects, depending on the behaviour of the dark energy fluid. Additionally, Zeng \& Gao (2005) and Manera \& Mota (2006) explore the effect of the different non-linear evolution on the predicted number counts for massive dark-matter halos (galaxy clusters), finding several significant effects. They discover, in particular, that the number counts of massive structures increase if small-scale clustering of dark energy is allowed, while they decrease if the amount of dark matter coupled to dark energy grows.

One highly sensitive way, which is interesting due to its non-linearity, to probe the massive end of the cluster population is the strong lensing effect. Although the issue is still controversial (Bartelmann et al. 1998; Meneghetti et al. 2000, 2003a; Wambsganss et al. 2004; Dalal et al. 2005; Li et al. 2005; Hennawi et al. 2005), it seems to be at least difficult within the $\Lambda \mathrm{CDM}$ model to reproduce the observed abundance of stronglensing events in cluster cores, the so-called gravitational arcs. Arcs in clusters at high redshift (Hasinger et al. 1998; Thompson et al. 2001; Zaritsky \& Gonzalez 2003; Gladders et al. 2003) are similarly puzzling because they indicate that even clusters at $z \gtrsim 1$ can already be concentrated and massive enough to be strong gravitational lenses for a source population that is not too distant from them.

Dynamical activity in galaxy clusters has been identified as highly important for their strong-lensing abilities (Bartelmann et al. 1995; Meneghetti et al. 2003b; Torri et al. 2004; Fedeli et al. 2006). The enhancement of the gravitational tidal (shear) field while clusters are merging with massive halos can transiently, but substantially, increase their strong-lensing cross sections. As much as about half of the total optical depth for strong cluster lensing may be contributed by merging clusters. The effect is strong because mergers can turn clusters into strong lenses that would otherwise fall below the threshold because they are not massive or compact enough. Major cluster mergers thus open the huge, exponentially rising reservoir of moderately massive clusters for strong lensing.

Cosmological models reconciling an appreciable cluster abundance at high redshift, and thus also a high level of dynamical cluster activity, with independent cosmological constraints - e.g. from the CMB - are thus particularly interesting in view of strong cluster lensing. Sufficiently detailed numerical 
simulations are costly and beyond the scope of a parameter study. Recently, Fedeli et al. (2006) developed a (semi-) analytic method for computing strong-lensing cross sections for galaxy clusters with and without taking cluster mergers into account. This method opens the way to systematically test a variety of cosmological models for their consequences for strong cluster lensing. We use it in this paper to study the statistics of strong cluster lensing in two exemplary cosmologies with early dark energy and compare them to the $\Lambda \mathrm{CDM}$ model and a model with a constant equation-of-state parameter $w>-1$. We do not consider any other aspects related to the dark-energy fluid behaviour, such as small-scale clustering or coupling to dark matter.

Section 2 reviews the extended Press-Schechter theory as it will be needed later, and Sect. 3 summarises the cosmological models used. The construction of merger trees and the computation of strong-lensing cross sections are described in Sects. 4 and 5. Section 6 outlines expectations, Sect. 7 quantifies the results, and Sect. 8 summarises and concludes the paper.

\section{Press-Schechter theory}

We begin by reviewing the basic features of the excursionset approach to the derivation of the Press-Schechter (Press \& Schechter 1974) mass function and the conditional mass function of virialised dark-matter halos. Comprehensive treatments can be found in Bond et al. (1991) and Lacey \& Cole (1993). The central physical quantity is the primordial Gaussian densityfluctuation field $\delta(\boldsymbol{x})$, filtered on a scale $R$ corresponding to a mass $M$. The filtered field $\delta_{M}(\boldsymbol{x})$ remains a Gaussian random field whose variance $S=S(M)$ is a monotonically decreasing function of mass. If the filter is a top-hat function in Fourier space, $\delta_{M}(\boldsymbol{x})$ at a fixed location $\boldsymbol{x}$ performs a random walk as a function of $M$ and thus of scale $R$.

When $\delta_{M}(\boldsymbol{x})$ rises above a critical, redshift-dependent threshold $\delta_{\mathrm{c}}(z)$, a halo of mass $M$ is expected to form at the location $\boldsymbol{x}$ at redshift $z$. The threshold $\delta_{\mathrm{c}}(z)$ is usually obtained from the spherical collapse model by linearly extrapolating the initial overdensity to the time when the collapsing halo reaches virial equilibrium. The problem of a random walk with a fixed absorbing barrier is solved in Chandrasekhar (1943) and leads, under the previous assumptions, to the mass function

$n(M, z)=\frac{\rho_{\mathrm{m}, 0}}{M} \frac{\delta_{\mathrm{c}}(z)}{\sqrt{2 \pi} S^{3 / 2} D_{+}(z)}\left|\frac{\mathrm{d} S}{\mathrm{~d} M}\right| \exp \left[-\frac{\delta_{\mathrm{c}}(z)^{2}}{2 S D_{+}(z)^{2}}\right]$.

This mass function is defined such that $n(M, z) \mathrm{d} M$ is the comoving number density of structures with mass between $M$ and $M+\mathrm{d} M$ at redshift $z$, where $\rho_{\mathrm{m}, 0}$ is the mean matter density at the present time. Unlike common practice, we explicitly introduced the linear growth factor $D_{+}(z)$ instead of incorporating it into the critical overdensity in order to emphasise that the redshift dependence in $\delta_{\mathrm{c}}(z)$ is exclusively due to the evolution of the spherical collapse model with redshift. In an Einstein-de Sitter universe, $\delta_{\mathrm{c}} \approx 1.686$ is a constant that evolves only subtly in a $\Lambda \mathrm{CDM}$ universe, but it changes considerably in the cosmological models with early dark energy. We thus have to account for its redshift evolution.

The formalism sketched so far can easily be extended to construct the conditional mass function that quantifies the probability of a halo of a given mass $M_{0}$ at a given redshift $z$ having a progenitor of a lower mass $M_{\mathrm{p}}$ at a higher redshift $z+\Delta z$. Since the variance of the density field $\delta_{M}(z)$ filtered on a scale corresponding to a given mass $M$ decreases monotonically with $M$,
Table 1. Cosmological parameters of the four models used in the present work.

\begin{tabular}{lllll}
\hline \hline & EDE1 & EDE2 & $w_{\mathrm{de}}=-0.8$ & $\Lambda$ CDM \\
\hline$\sigma_{8}$ & 0.82 & 0.78 & 0.80 & 0.84 \\
$h$ & 0.67 & 0.62 & 0.65 & 0.65 \\
$\Omega_{\mathrm{m}, 0}$ & 0.33 & 0.36 & 0.30 & 0.30 \\
$\Omega_{\mathrm{de}, 0}$ & 0.67 & 0.64 & 0.70 & 0.70 \\
\hline
\end{tabular}

this is equivalent to the probability that a halo of variance $S\left(M_{0}\right)$ at a given redshift had a higher variance $S\left(M_{\mathrm{p}}\right)$ at a higher redshift. This probability is given by (Lacey \& Cole 1993)

$K(\Delta S, \Delta \omega)=\frac{1}{\sqrt{2 \pi}} \frac{\Delta \omega}{\Delta S^{3 / 2}} \exp \left[-\frac{\Delta \omega^{2}}{2 \Delta S}\right]$

where $\Delta S=S\left(M_{\mathrm{p}}\right)-S\left(M_{0}\right)$, and $\Delta \omega$ represents the redshift step considered,

$\Delta \omega=\frac{\delta_{\mathrm{c}}(z+\Delta z)}{D_{+}(z+\Delta z)}-\frac{\delta_{\mathrm{c}}(z)}{D_{+}(z)}$

In other words, Eq. (2) gives the probability of a dark-matter halo undergoing a change in variance $\Delta S$ due to hierarchical accretion in the redshift interval $\Delta z$.

If we want the probability that the halo of mass $M_{0}$ has a progenitor corresponding to a change in variance lower than $\Delta S$ within the same redshift interval, we simply have to integrate the above equation, obtaining the cumulative probability distribution

$J(\Delta S, \Delta \omega)=\int_{0}^{\Delta S} K(\Delta \zeta, \Delta \omega) \mathrm{d} \Delta \zeta=\operatorname{erfc}\left(\frac{\Delta \omega}{\sqrt{2 \Delta S}}\right)$,

where

$\operatorname{erfc}(x) \equiv \frac{2}{\sqrt{\pi}} \int_{x}^{\infty} \mathrm{e}^{-t^{2}} \mathrm{~d} t$

is the complementary error function. Equation (4) is just the probability of the mass of the progenitor $M_{\mathrm{p}}$ being larger than the mass corresponding to the variance $\Delta S-S\left(M_{0}\right)$.

\section{Dark-energy models}

Dark energy generalises Einstein's cosmological constant, replacing it by a term varying with redshift in Friedmann's equation. Viable model universes with dark energy must be adapted to comply with present-day observational data. Many models motivated by elementary-particle physics introduce dark energy as a scalar field (called cosmon or quintessence, see for instance Wetterich 1988; Peebles \& Ratra 1988, 2002; Brax \& Martin 2000), whose pressure and energy density are related by the perfect-fluid equation of state

$P_{\mathrm{de}}=w_{\mathrm{de}} \rho_{\mathrm{de}} c^{2}$.

The parameter $w_{\mathrm{de}}$ is typically a function of cosmic time or redshift. A cosmological constant has $w_{\mathrm{de}}=-1$ at all redshifts. Different models give rise to different (often not analytic) shapes for the function $w_{\mathrm{de}}(z)$, and there are various ways to parameterise a chosen quintessence model.

Negative pressure at all times implies that the dark-energy density parameter $\Omega_{\mathrm{de}}(z)$ will fall to zero for increasing redshift. If, however, the equation-of-state parameter $w$ is allowed to rise above zero, models can be constructed in which $\Omega_{\mathrm{de}}(z)$ 
settles at a small positive value. The presence of a non-vanishing dark-energy contribution in early epochs of the cosmic evolution can have many interesting consequences on the CMB temperature fluctuations, the geometry and the age of the Universe, and the linear and non-linear aspects of structure formation. Darkmatter halos on all mass scales may form substantially earlier (Bartelmann et al. 2005, see also Dolag et al. 2004), potentially causing large effects on the statistics of strong-lensing events (cf. Bartelmann et al. 2003).

Wetterich (2004) proposes a useful characterisation of cosmological models with early dark energy that makes use of only three parameters: the present density parameter in the dark en$\operatorname{ergy} \Omega_{\mathrm{de}, 0}$, the present equation-of-state parameter $w_{\mathrm{de}, 0}$, and an average value for the dark energy parameter at early (structure formation) times,

$\bar{\Omega}_{\mathrm{de}, \mathrm{sf}} \equiv-\left(\ln a_{\mathrm{eq}}\right)^{-1} \int_{\ln a_{\mathrm{eq}}}^{0} \Omega_{\mathrm{de}}(a) \mathrm{d} \ln a$

where $a_{\mathrm{eq}}$ is the scale factor at matter-radiation equality.

For sufficiently low $w_{\mathrm{de}, 0}$, such phenomenological early darkenergy models reproduce the accelerated cosmic expansion in the present-day Universe similar to cosmological-constant models and can thus be arranged to agree with low-redshift observations. If $\bar{\Omega}_{\mathrm{de}, \mathrm{sf}}$ is small enough, they can also reproduce the CMB temperature fluctuations. We investigate here the same two early dark-energy models as in Bartelmann et al. (2005), which have $\bar{\Omega}_{\mathrm{de}, \mathrm{sf}}=0.04$, spectral indices for the primordial densityfluctuation power spectrum of $n=1.05$ (model EDE1 henceforth) and of $n=0.99$ (hereafter model EDE2). For comparison, we also include a model with a constant equation-of-state parameter $w=-0.8$ and a conventional $\Lambda$ CDM model for reference.

The values of the other cosmological parameters, such as the present-day matter-density parameter $\Omega_{\mathrm{m}, 0}$, the dimension-less Hubble constant $h$, and the normalisation of the power spectrum expressed by $\sigma_{8}$, were determined so as to match the power spectrum of the CMB temperature fluctuations (Spergel et al. 2003, 2006), constraints from the large-scale structure of the Universe (Tegmark et al. 2004), and observations of type-Ia supernovae (Riess et al. 2004). The values characterising the four cosmological models used in this paper are listed in Table 1, while Fig. 1 shows the redshift evolution of the equation-of-state parameter in these cosmologies.

As can be noted, the early dark-energy models approach the cosmological-constant scenario very close at very low redshifts.

\section{Merger trees}

We now proceed to use the extended Press-Schechter formalism summarised in Sect. 2 for a Monte-Carlo realisation of merger trees. The procedure is quite straightforward, and we refer to Somerville \& Kolatt (1999) for a detailed discussion and to Randall et al. (2002) and Cassano \& Brunetti (2005) for some applications.

\subsection{Monte-Carlo simulations}

Consider a halo of mass $M_{0}$ at the present time $(z=0)$. If we draw a random number $r$ in the interval $[0,1]$ and solve the equation $J(\Delta S, \Delta \omega)-r=0$, we draw a value for the change in the variance corresponding to the halo compliant with the merger rate (4).

Given the variance $S\left(M_{0}\right)$ of the halo's original mass, we obtain a new value of the variance and convert it to a new mass

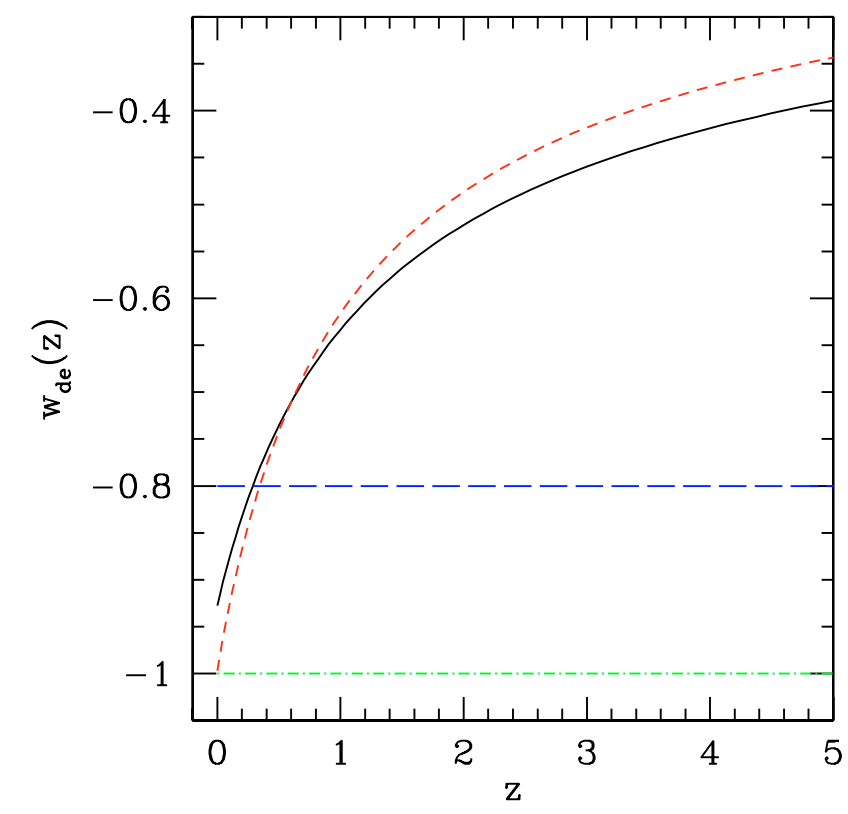

Fig. 1. The redshift evolution of the equation-of-state parameter for the four cosmological models used in this paper. These are a $\Lambda \mathrm{CDM}$ model (green dashed-dotted line), a model with constant $w_{\text {de }}=-0.8$ (blue long-dashed line), and two early dark-energy models with different spectral indices for the primordial density fluctuations (the black solid line represents EDE1 and the red-dashed line EDE2).

that is the mass of the progenitor $M_{\mathrm{p}}$. If we choose a sufficiently small time interval, we can assume that the entire change in the halo's mass is due to a unique, binary merging process with another halo of mass $\Delta M=M_{0}-M_{\mathrm{p}}$. If we repeat this process for earlier progenitors at subsequent redshift steps, we obtain the merger history of the original halo up to a given redshift. At the end of this procedure, we have obtained the value of the halo's mass and that of its progenitors for each redshift step, i.e. a merger tree.

The choice of the time interval needs some care. It has to be small to justify the assumption of binary mergers, but not too small to avoid the results being dominated by numerical noise. Following the rule-of-thumb given by Lacey \& Cole (1993), we use a time step such that

$\Delta \omega=\sqrt{\frac{\mathrm{d} S\left(M_{0}\right)}{\mathrm{d} M} \Delta M_{\mathrm{c}}}$

(see also Somerville \& Kolatt 1999) where $\Delta M_{\mathrm{c}}$ is the mass of the smallest sub-halo required to be resolved individually. If $M_{\mathrm{p}}$ or $\Delta M$ fall below $\Delta M_{\mathrm{c}}$, the process does not represent an individual merger, but smooth accretion. It follows from the above expression that the lower initial masses $M_{0}$ require larger time steps.

A set of Monte-Carlo realisations of merger trees is successful if the population of structures that it produces agrees with the theoretical mass function at any given redshift. As Somerville \& Kolatt (1999) pointed out, this is not strictly so if we consider only binary mergers and smooth accretion as we are doing here. Several authors (Benson et al. 2005) argued that this may be due to an intrinsic inconsistency in the extended PressSchechter formalism, and Somerville \& Kolatt (1999) suggest that the problem can be mitigated considering multiple mergers and smooth accretion. Nonetheless, the difference between the halo-mass distributions following from the merger-tree simulations and expected from the mass function is significant only 


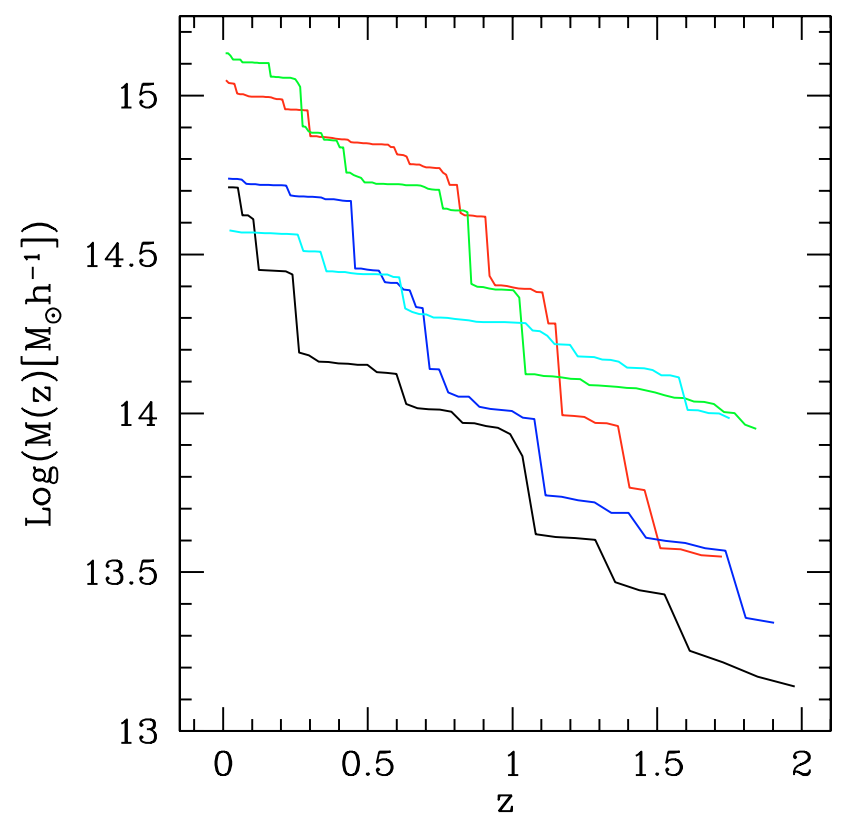

Fig. 2. Exemplary merger histories for five dark-matter halos randomly selected from our sample in a $\Lambda \mathrm{CDM}$ universe. The merger histories are extended up to the source redshift for each individual halo.

at redshifts beyond our interest, and several tests confirmed the good agreement between the two halo-mass distributions.

\subsection{Our sample}

We consider a sample of $\mathcal{N}=500$ dark-matter halos whose present-day masses are uniformly distributed within $M_{\text {inf }}=$ $10^{14} h^{-1} M_{\odot}$ and $M_{\text {sup }}=2.5 \times 10^{15} h^{-1} M_{\odot}$. It is plausible that structures with mass below $M_{\text {inf }}$ at $z=0$ do not contribute appreciably to the total lensing efficiency (see the discussion in Fedeli et al. 2006). For each halo, we compute the appropriate time step from (8) and split it into two progenitor halos. Then, we proceed with the more massive progenitor as the starting point for the next step. We repeat this procedure until the redshift exceeds the source redshift $z_{\mathrm{s}}$ (which is chosen individually for each halo in the sample, see Sect. 4 for details) or the mass of the halo falls below $\Delta M_{\mathrm{c}}$.

We show in Fig. 2 the merger histories (that is, the evolution of mass with redshift) of five halos selected from our sample of 500 halos for a $\Lambda \mathrm{CDM}$ model. Sudden discontinuities in the mass are evident, each of which corresponds to a merger between the main halo and a massive sub-halo.

\section{Strong-lensing statistics}

In order to compute the efficiency of dark-matter halos as strong cluster lenses, specifically for producing long and thin arcs, we model each halo as an NFW (Navarro et al. 1996, 1997) density profile with an elliptically distorted lensing potential. Following Meneghetti et al. (2003b), we adopt an ellipticity for the isopotential contours equal to $e=0.3$ for all halos. Deflectionangle maps for such a lens model can be calculated analytically (Bartelmann 1996). We then use the fast, semi-analytic method developed by Fedeli et al. (2006) to compute the cross sections. We describe only its main features here and refer the reader to the cited paper for details.
The lens equation

$y(x)=x-\alpha(x)$

relates the (dimensionless) position $\boldsymbol{y}$ of a point source to the positions $\boldsymbol{x}$ of its images. In the single lens-plane case we consider here, the Jacobian matrix of the mapping (9) is symmetric, hence can be diagonalised through an orthogonal transformation. The eigenvalues of the Jacobian give the distortions of the image along the independent directions of the corresponding eigenvectors. Thus, the ratio of the eigenvalues determines the length-to-width ratio of the images of point-like sources.

Based upon this consideration, the cross section for gravitational arcs with length-to-width ratio exceeding a given threshold can be evaluated as the integral of the inverse of the magnification (the Jacobian determinant) over the region of the lens plane where the ratio of the eigenvalues is higher than the threshold. The region of integration will of course be a stripe surrounding the critical curves of the mapping. However, this calculation ignores the fact that real sources are both extended and noncircularly shaped, which together enlarge the cross section compared to point sources.

Thus, to obtain the length-to-width ratio of extended sources, we convolve the ratio of the eigenvalues with a step function of width equal to the source size (assumed to be $0.5^{\prime \prime}$ in radius), and we use the elegant formalism developed in Keeton (2001) to account for source ellipticities, which are randomly drawn from a flat distribution between 0.5 and 1 . This method allows us to calculate the cross section $\sigma_{\mathrm{d}}$ for arcs with a length-to-width ratio exceeding some given threshold $d$. We choose $d=7.5$ here and show one plot with $d=10$ for comparison later.

These semi-analytic cross sections are in excellent agreement with the results from fully numerical ray-tracing simulations. Moreover, their computation is substantially faster since the method does not require costly operations such as finding all images for every source and refining the source distribution near caustics on an adaptive grid.

We calculate cross sections both ignoring and accounting for merger processes that transiently increase the lensing efficiency. When a merger with a sub-halo of mass larger than $5 \%$ of the main halo's mass occurs, we model the interaction as follows. The two clumps of dark matter approach each other at a constant speed starting from an initial distance set to the sum of their virial radii, $r_{\mathrm{v}, 1}+r_{\mathrm{v}, 2}$. The process concludes when the profiles overlap completely, i.e. when their centres coincide in projection, and its duration is set to the dynamical timescale

$T_{\mathrm{dyn}}=\sqrt{\frac{\left(r_{\mathrm{v}, 1}+r_{\mathrm{v}, 2}\right)^{3}}{G\left(M_{1}+M_{2}\right)}}$.

For a fixed and constant source redshift $z_{\mathrm{s}}$, we can compute the optical depth $\tau_{\mathrm{d}}\left(z_{\mathrm{s}}\right)$ once the strong-lensing cross sections for each halo at all redshift steps between the observer $(z=0)$ and the sources $\left(z=z_{\mathrm{s}}\right)$ are known. It is

$\tau_{\mathrm{d}}\left(z_{\mathrm{s}}\right)=\frac{1}{4 \pi D_{\mathrm{s}}^{2}} \int_{0}^{z_{\mathrm{s}}} \int_{0}^{\infty} N(M, z) \sigma_{\mathrm{d}}\left(M, z, z_{\mathrm{s}}\right) \mathrm{d} M \mathrm{~d} z$

where $D_{\mathrm{s}}$ is the angular-diameter distance to the source sphere, and $N(M, z) \mathrm{d} z$ is the number of structures with mass $M$ included in the shell between redshift $z$ and $z+\mathrm{d} z$. However, in order to account for the source redshift distribution, we randomly assigned to each dark-matter halo an individual source redshift $z_{\mathrm{s}, i}, i=1, \ldots, \mathcal{N}$, drawn from the redshift distribution of faint 


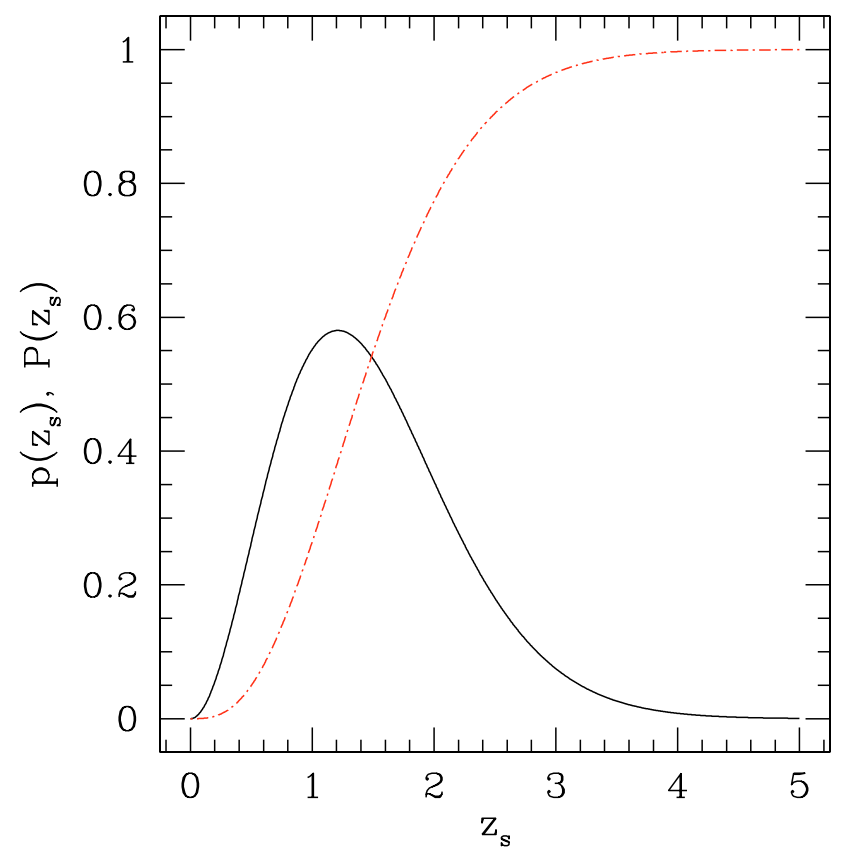

Fig. 3. The differential (black solid line) and cumulative (red dashdotted line) source-redshift distributions given by Eq. (12).

blue galaxies given in (Smail et al. 1995; see also Bartelmann \& Schneider 2001)

$p\left(z_{\mathrm{s}}\right)=\frac{\beta}{z_{0}^{3} \Gamma(3 / \beta)} z_{\mathrm{s}}^{2} \exp \left[-\left(\frac{z_{\mathrm{s}}}{z_{0}}\right)^{\beta}\right]$.

The parameters $z_{0}$ and $\beta$ define the average redshift and the steepness of the high-redshift tail of the distribution, respectively. In this work, we used the conventional values $z_{0}=1$ and $\beta=3 / 2$. Given this choice, the distribution peaks at $z_{\mathrm{m}} \simeq 1.21$. Figure 3 shows this distribution together with its cumulative function defined by

$P\left(z_{\mathrm{s}}\right)=\int_{0}^{z_{\mathrm{s}}} p(z) \mathrm{d} z$

Using the distribution (12), we can define the average optical depth as

$$
\begin{aligned}
\bar{\tau}_{\mathrm{d}} & =\int_{0}^{\infty} \tau_{\mathrm{d}}\left(z_{\mathrm{s}}\right) p\left(z_{\mathrm{s}}\right) \mathrm{d} z_{\mathrm{s}} \\
& =\int_{0}^{\infty}\left[\int_{0}^{z_{\mathrm{s}}} \int_{0}^{\infty} \sigma_{\mathrm{d}}\left(M, z, z_{\mathrm{s}}\right) N(M, z) \frac{\mathrm{d} M \mathrm{~d} z}{4 \pi D_{\mathrm{s}}^{2}}\right] p\left(z_{\mathrm{s}}\right) \mathrm{d} z_{\mathrm{s}} .
\end{aligned}
$$

Since each halo in our study is characterised by a source redshift randomly drawn from the distribution (12), we can omit the weighting with $p\left(z_{\mathrm{s}}\right)$ when we discretise the integral over source redshift in (14). However, this is not possible for the mass integration, since the masses of the halos are randomly drawn from a uniform distribution, which requires the weighting with the halo mass function.

The source-redshift distribution $p\left(z_{s}\right)$ formally extends to an infinite source redshift, but obviously this is not true in reality. We set the maximum source redshift to $z_{\max }=7.5$. As Fig. 3 shows, the probability of finding a source at this redshift can safely be neglected. Since we operate on a discrete sample of $\mathcal{N}$

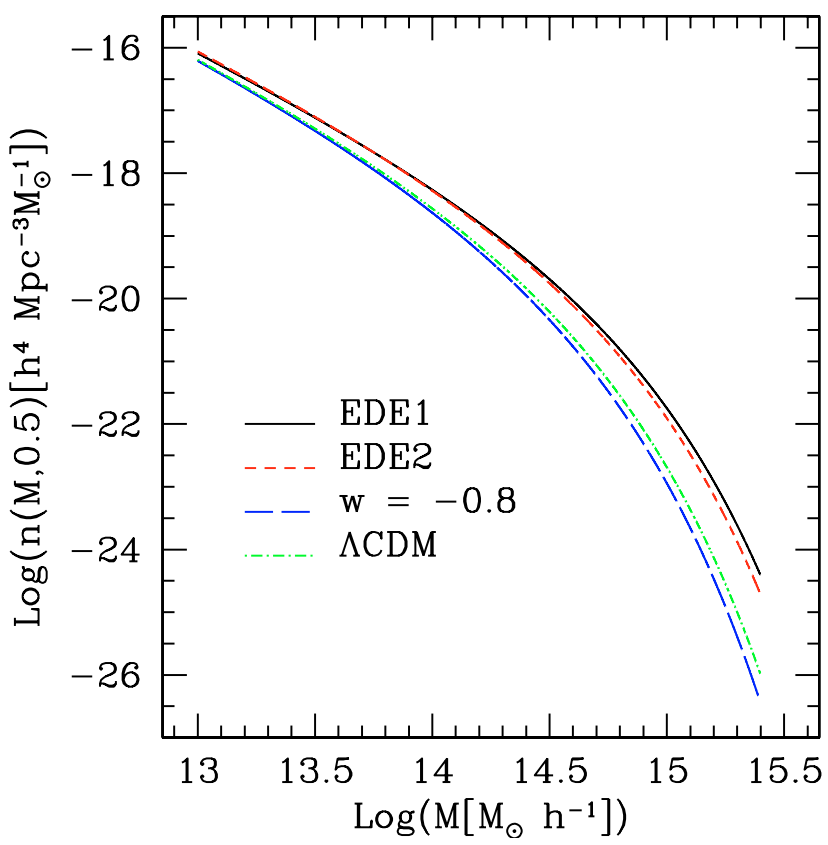

Fig. 4. The mass function for dark-matter halos in the mass range $\left[10^{13}, 2.5 \times 10^{15}\right] h^{-1} M_{\odot}$ at redshift $z=0.5$ for the four cosmological models used in this paper, as labelled in the plot.

halos, each of which is characterised by a mass $M_{i}$ and a source redshift $z_{\mathrm{s}, i}$, we can rewrite (14) as

$\bar{\tau}_{\mathrm{d}}=\int_{0}^{z_{\max }}\left[\sum_{i=1}^{\mathcal{N}-1} \frac{\sigma_{\mathrm{d}}\left(M_{i}, z, z_{\mathrm{s}, i}\right)}{4 \pi D_{\mathrm{s}, i}^{2}} \int_{M_{i}}^{M_{i+1}} N(M, z) \mathrm{d} M\right] \mathrm{d} z$.

The integrand of this equation is the optical depth per unit redshift, i.e. the contribution to the optical depth from halos at different redshifts, accounting for the source-redshift distribution

$t_{\mathrm{d}}(z)=\sum_{i=1}^{N-1} \frac{\sigma_{\mathrm{d}}\left(M_{i}, z, z_{\mathrm{s}, i}\right)}{4 \pi D_{\mathrm{s}, i}^{2}} \int_{M_{i}}^{M_{i+1}} N(M, z) \mathrm{d} M$.

This will be the central quantity in our strong-lensing analysis.

\section{Expectations}

Before turning to the results, it is useful to evaluate the expectations in order to gain a better understanding of the problem. As shown by Bartelmann et al. (2005), the formation of nonlinear cosmic structures occupies a larger redshift range in early darkenergy cosmological models. Structures form earlier and the formation process lasts longer. This increases the merger probability for a given halo at high redshift, as well as the total number of structures of a given mass that are found at a given redshift. Figure 4 shows the mass function (1) at a fixed redshift $z=0.5$ for the four cosmological models used in this paper.

Evidently, the mass function is lowest for a $\Lambda \mathrm{CDM}$ model and only slightly higher for a model with constant equation-ofstate parameter $w=-0.8$. It is highest (by up to an order of magnitude at the high-mass tail) for the two early dark-energy models. This reflects the different halo-formation histories in different cosmologies. In the EDE1 and EDE2 models, structure formation begins earlier, hence at a given (suitably low) redshift, the abundance of halos is higher.

In Fig. 5, we show the merger rate for a halo of mass $M=10^{14} h^{-1} M_{\odot}$ and a sub-halo of mass $M / 2$ as a function of 


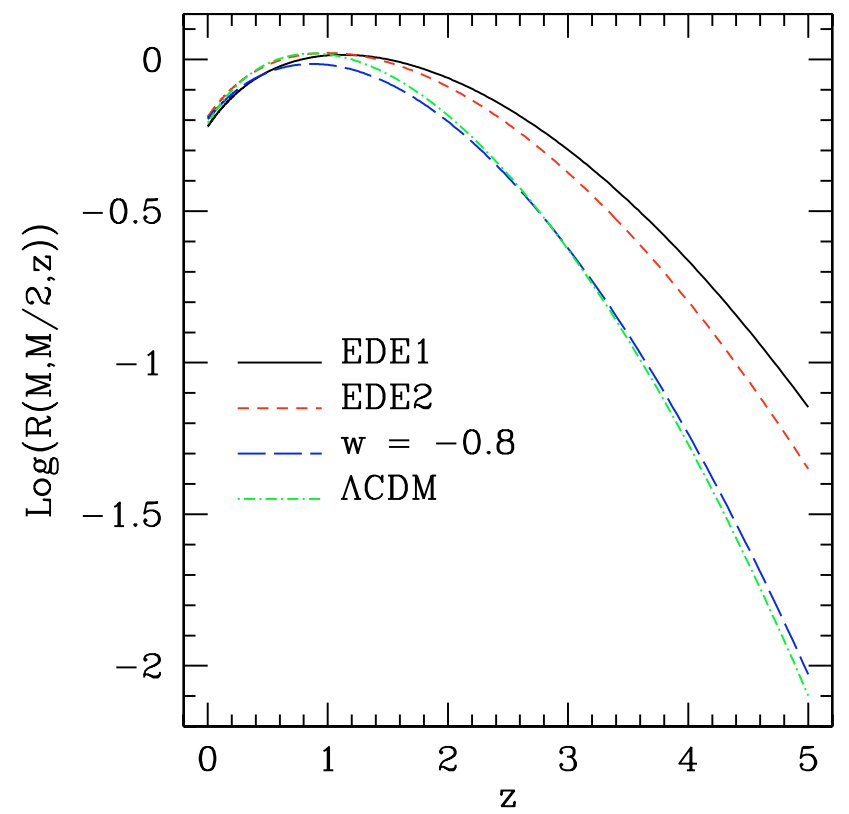

Fig. 5. The probability that a dark-matter halo of mass $M=10^{14} h^{-1} M_{\odot}$ merges with a sub-halo of mass $M / 2$ as a function of redshift per unit logarithmic mass of the merging sub-halo and per unit logarithmic cosmic time. Results are shown for all four cosmological models considered here, as labelled in the plot.

redshift. By merger rate, we mean the probability that a halo of mass $M$ merges with a sub-halo of mass $M / 2$ at redshift $z$ per unit logarithmic sub-halo mass and per unit logarithmic cosmic time. It can be obtained as the appropriate limit of the conditional probability distribution Eq. (2).

Regarding the merger rate, we also note the difference between the behaviour of early dark-energy models and of models with a constant equation-of-state parameter. At high redshift, the early dark-energy merger rate is significantly higher than for the other two models, but becomes essentially the same below redshift $\sim 1.2$.

This can again be understood in terms of the different dynamics of structure formation. Keeping the mass of the sub-halo fixed, we expect more halos of such mass to be available at high redshift with which the main halo can merge, because structure formation begins earlier in early dark-energy models. On the other hand, structure growth begins later in models with a constant equation-of-state parameter and proceeds more rapidly. Thus, at a sufficiently low redshift, the abundance of such halos equals that in early dark-energy models, giving rise to an almost identical merger rate.

It is worth emphasising here that the differences shown between the different cosmological models are also due, in part or mainly, to the different normalisation $\sigma_{8}$ of the power spectrum, which is chosen to make the models agree with the CMB observations.

Recalling that the source-redshift distribution peaks at redshift $\sim 1.2$, we expect the different merger rates to have little influence on the optical depth. On the other hand, since the optical depth is essentially an average of the cross section of different halos weighted by their relative abundances, we expect the difference in the mass function to severely affect the strong-lensing statistics. In early dark-energy models, the optical depth per unit redshift should exceed those in the $\Lambda \mathrm{CDM}$ model and the model with a constant equation-of-state parameter of $w=-0.8$. We show in the next section how well this expectation is satisfied.

\section{Results}

We discuss now the expected behaviour of the optical depth per unit redshift (see definition in Sect. 4) in the different darkenergy cosmologies considered in this work. The occurrence of gravitational arcs is highly sensitive to the abundance and internal structure of galaxy clusters, which in turn depends on the linear and non-linear evolution of density fluctuations. We thus expect that the presence and behaviour of dark energy can affect it.

We show the optical depth per unit redshift in Fig. 6 for arcs with a length-to-width ratio exceeding $d=7.5$, obtained for each of our four cosmological models. For model EDE1, we also show the result for $d=10$. The optical depths accounting for and ignoring halo mergers are compared. As expected, the lensing efficiency vanishes near the observer and approaching the source redshift because of the geometrical drop in lensing efficiency.

Cluster mergers increase the optical depth per unit redshift, and thus also the total optical depth, factors up to 2 or 3 in all dark-energy models. The enhancement due to mergers appears more uniform than obtained by Fedeli et al. (2006). This is due to the sample used here that is more than one order of magnitude larger and to the much higher time resolution adopted (up to $10^{-2}$ in redshift). Quite obviously, increasing the length-towidth threshold decreases the lensing efficiency, but the features caused by merger processes remain qualitatively the same.

The main result is that mergers enhance the lensing efficiency by about the same amount for each model because the merger rate is almost the same in the redshift range relevant to strong cluster lensing. However, note that the absolute value of the optical depth per unit redshift is higher in early dark-energy models, which is seen better in Figs. 7 and 8. There, we compare the optical depth per unit redshift for arcs with length-to-width ratios exceeding $d=7.5$ in the four cosmologies, accounting for (Fig. 7) and ignoring cluster mergers (Fig. 8).

This effect was also expected because of the difference in the abundance of halos of a given mass in various cosmological models. These figures show that, both with and without the effect of halo mergers, the lensing optical depth per unit redshift is higher by factors up to $\sim 3$ in early dark-energy models compared to the other models. At redshifts above $\sim 0.5$, the lensing efficiency for the model with a constant $w=-0.8$ is slightly smaller than in the $\Lambda \mathrm{CDM}$ model because the abundance of halos is also slightly lower (see Fig. 4). A similar difference appears between the EDE1 and EDE2 models. This is due to the fact that the normalisation of the power spectrum is higher in the first than in the second, causing a higher abundance of clusters.

An effect that we also recognise in these plots is that, in cosmologies with early dark energy, the optical depth per unit redshift rises and already reaches a significant level at relatively high redshift, while it is still negligible in a $\Lambda$ CDM model. As discussed before, the models alternative to $\Lambda \mathrm{CDM}$ that we have studied here have a larger fraction of structures at high redshift, causing this earlier and larger contribution to the strong-lensing efficiency.

Further detail on this aspect is provided by Fig. 9. In its top panels, it shows the cumulative optical depth per unit redshift, which we can write as

$C_{\mathrm{d}}(z)=\int_{z}^{z_{\max }} t_{\mathrm{d}}\left(z^{\prime}\right) \mathrm{d} z^{\prime}$,

normalised to the $\Lambda \mathrm{CDM}$ case. Its increase towards high redshift emphasises directly how the lensing efficiency already drops at 

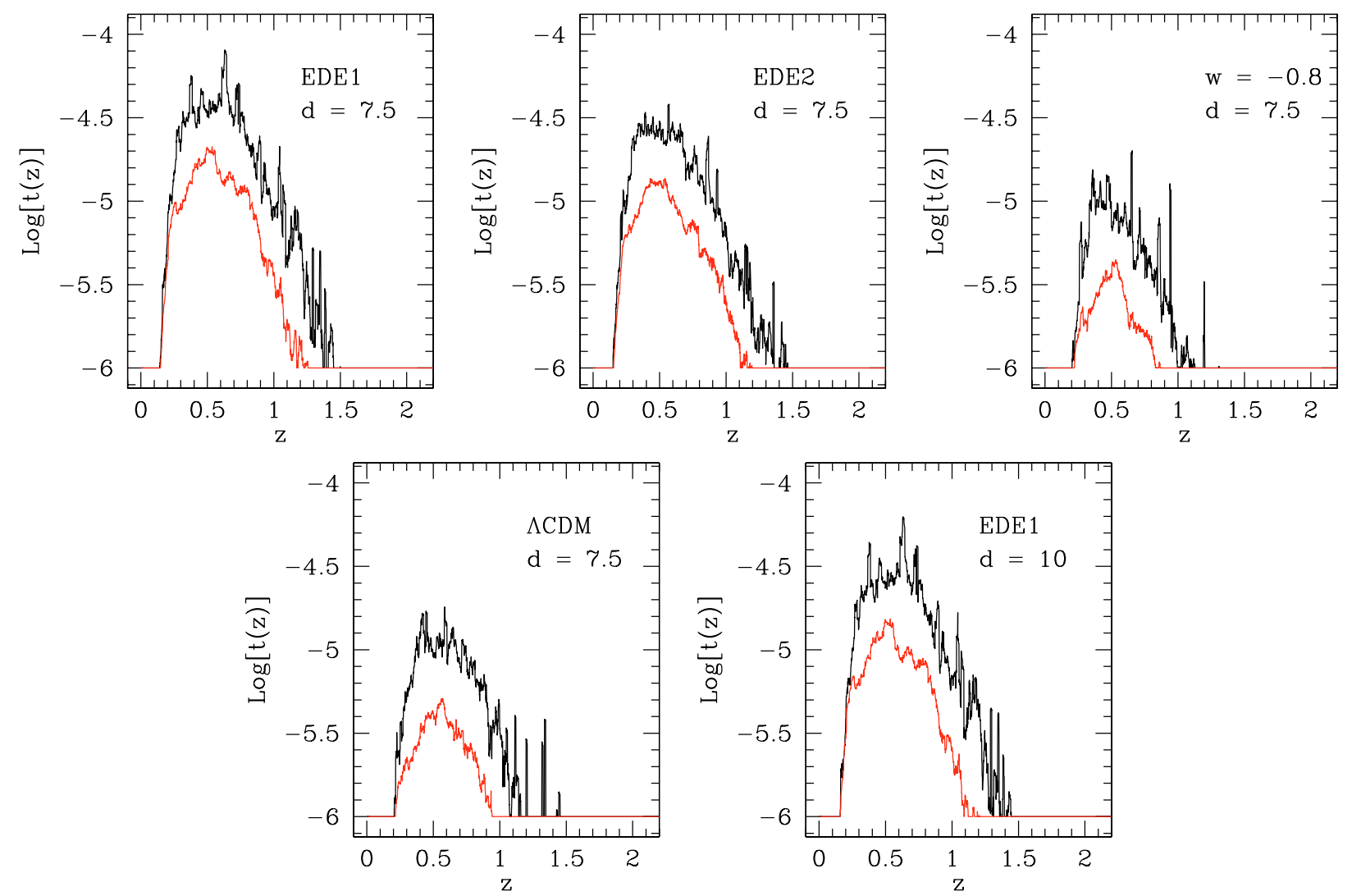

Fig. 6. The logarithm of the optical depth per unit redshift for arcs with length-to-width ratio exceeding $d=7.5$ for each of the cosmological models studied here (top and bottom-left panels), and for $d=10$ in the model EDE1 (bottom-right panel). Black curves show the optical depths obtained including halo mergers with sub-halos, while red curves are obtained after ignoring the effect of halo interactions.

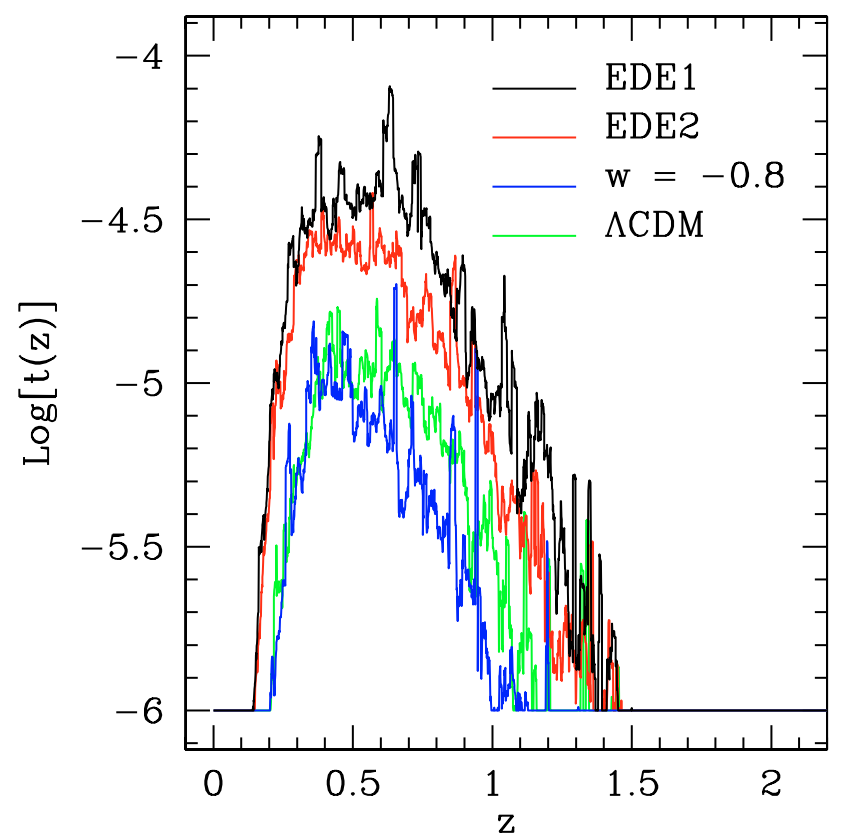

Fig. 7. The logarithm of the optical depth per unit redshift for arcs with length-to-width ratio exceeding $d=7.5$ obtained for the four darkenergy models considered here. The lensing efficiency shown here takes transient boosts by cluster mergers into account.

lower redshift in a $\Lambda \mathrm{CDM}$ Universe with respect to the (early) dark-energy cosmologies. The bottom panels show the cumulative optical depth per unit redshift normalised to the present

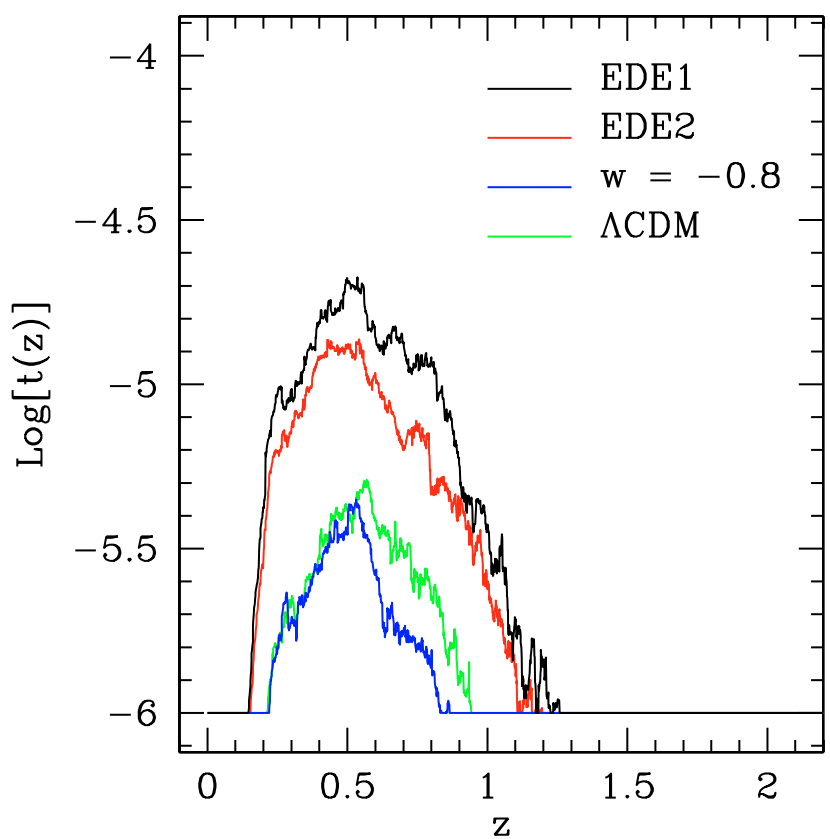

Fig. 8. Similar to Fig. 7, but ignoring the effect of cluster mergers on the lensing efficiency.

value in the $\Lambda \mathrm{CDM}$ model, $C_{\mathrm{d}, \Lambda \mathrm{CDM}}(0)=\bar{\tau}_{\mathrm{d}, \Lambda \mathrm{CDM}}$. This illustrates the same effect in a different way. For instance, we see that the cumulative optical depth per unit redshift in the EDE1 model already reaches the same value $C_{\mathrm{d}, \Lambda \mathrm{CDM}}(0)$ at $z \approx 0.8$, 

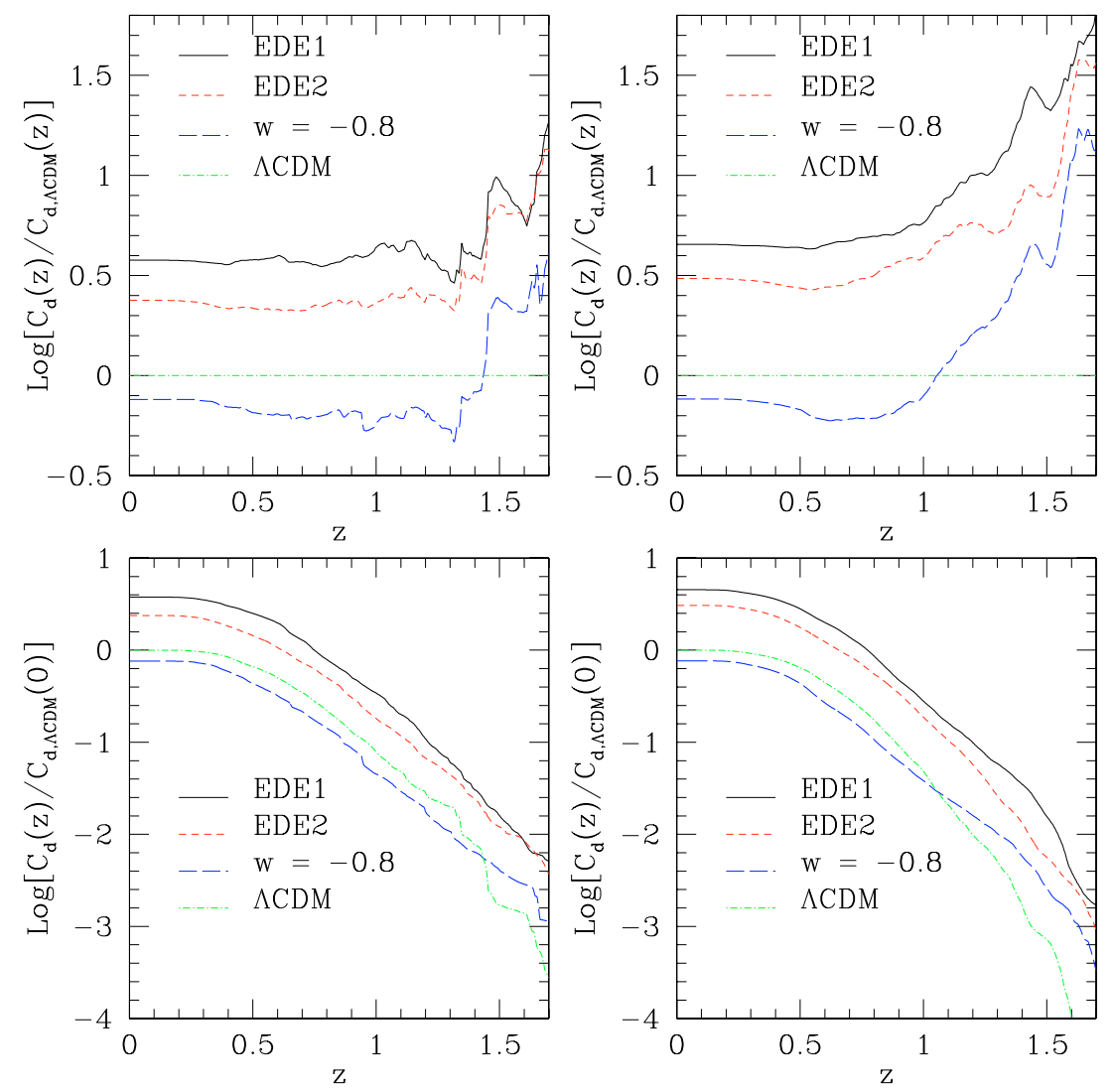

Fig. 9. Top panels: Logarithm of the cumulative optical depth per unit redshift, normalised to its value in a $\Lambda$ CDM universe. Curves representing the four cosmologies studied in this work are shown, as labelled in the plot. Bottom panels: Logarithm of the cumulative optical depth per unit redshift, normalised to its value at present in a $\Lambda$ CDM universe. Left and right panels show results that include and ignore cluster mergers, respectively.

which $\Lambda \mathrm{CDM}$ reaches today. Conversely, the cumulative optical depth per unit redshift in the $\Lambda$ CDM case has already dropped by an order of magnitude by $z \approx 0.8$. Similarly, the EDE2 model reaches the total optical depth of the $\Lambda \mathrm{CDM}$ model at $z \approx 0.7$. In agreement with our earlier discussion, we note that this specific evolution does not depend on whether we take dynamical processes into account or not. The enhanced lensing efficiency in the high redshift tail may have stimulating consequences, as we shall discuss later.

The large spikes shown in Fig. 7 are obviously due to the variation in the lensing efficiency of galaxy clusters during mergers. Very small spikes also appear in Fig. 8, where dynamical processes are not taken into account. There, they stem from numerical effects, in particular, now our time resolution is very high and the number of halos is limited. Indeed, the spikes become larger well above redshift unity, where the number of contributing halos is reduced (remember that each halo is characterised by a different source redshift, drawn from a distribution that peaks around $z \approx 1.2$ ).

\section{Summary and discussion}

We have analysed the incidence of pronounced (long and thin) arcs in galaxy clusters in four dark-energy models. In particular, we considered two early dark-energy cosmologies in which the density parameter in dark energy at high redshift remains small and positive. We compared them to a model with constant equation-of-state parameter $w_{\mathrm{de}}=-0.8$ and a $\Lambda \mathrm{CDM}$ model for which $w_{\mathrm{de}}=-1$.
For each cosmological model, we used Monte-Carlo techniques to build up merger trees for a set of $\mathcal{N}=500$ cluster-sized dark-matter halos. By modelling each halo by an NFW density profile with elliptically distorted lensing potential and suitably accounting for cluster interactions during mergers, we calculated the optical depth per unit redshift both accounting for and ignoring cluster mergers. To this end, we also considered a realistic distribution for the source redshift.

We find that, in agreement with the results of Fedeli et al. (2006), cluster mergers enhance the occurrence of arcs by a factor between 2 and 3. This occurs in all the cosmological models we analysed, and the relative increase is approximately the same, because the cluster merger rates in the redshift ranges relevant for strong lensing (below $z \sim 1$ ) are almost identical (see the discussion in Sect. 6).

However, a potentially more important result is that the optical depth per unit redshift is larger by a factor of $\sim 3$ in early darkenergy models compared to the models with a cosmological constant or with a constant equation of state parameter $w_{\mathrm{de}}=-0.8$, while the differences between the last two are close to negligible. There is also a significant difference between the two early darkenergy models due to the fact that model EDE1 has a higher normalisation parameter $\sigma_{8}$ than EDE2 in order to agree with the CMB observations (cf. Table 1). Thus, halos form earlier in model EDE1. This is also demonstrated by Figs. 4 and 5. Moreover, the lensing efficiency already drops at a lower redshift in a $\Lambda C D M$ Universe than in the different dark-energy models. The optical depth per unit redshift has a significant high-redshift tail in early dark-energy cosmologies, while it is negligible otherwise. 
A main consequence of these results is that they indicate an appreciable difference in the incidence of long and thin gravitational arcs between the $\Lambda \mathrm{CDM}$ model and models with early dark energy. Therefore, arc statistics may provide an interesting way to investigate the reliability of these models, although the precise contribution of $\bar{\Omega}_{\mathrm{de}, \mathrm{sf}}$ will probably be better constrained using cluster counts in the $X$-ray or Sunyaev-Zel'dovich regimes, which suffer from lower systematics.

The presence of early dark energy, combined with the transient boosts due to cluster mergers, could help resolve the discrepancy between the predicted and observed abundances of gravitational arcs. Ever since Bartelmann et al. (1998) first pointed out this problem for a $\Lambda \mathrm{CDM}$ universe, much discussion has developed around it (Meneghetti et al. 2000, 2003b,a; Wambsganss et al. 2004; Li et al. 2005). At present, it seems that neither the internal structure of the lensing halos nor the redshift distribution of the sources can reconcile theory and observations. It has been shown here that the effects of early dark energy on structure growth interestingly point in the right direction. Similar conclusions were also drawn by Meneghetti et al. (2005), where the lensing efficiency of numerically simulated dark-matter halos in different dark energy cosmologies were analysed. Here the haloes are modelled in an analytical way, allowing a much higher mass and time resolution. Moreover, the dark energy models studied there were derived from SUGRA and Ratra-Peebles potentials (Peebles \& Ratra 2002), without an early component. In many aspects, our work is thus complementary to that of Meneghetti et al. (2005).

Finally, that the lensing efficiency in early dark-energy models is much higher at high redshift than in the $\Lambda \mathrm{CDM}$ case can be related to the recent unexpected discovery of the high incidence of giant arcs in high-redshift clusters (Gladders et al. 2003; Zaritsky \& Gonzalez 2003). Future searches for strong lensing in distant galaxy clusters may be promising for distinguishing between cosmological models other than the standard $\Lambda \mathrm{CDM}$, or at least for gaining a deeper understanding of the role of early dark energy.

Acknowledgements. We are grateful to M. Meneghetti and L. Moscardini for comments on the manuscript and useful discussions. This work was supported by the Collaborative Research Centre SFB 439 of the Deutsche Forschungsgemeinschaft and by the German Academic Exchange Service (DAAD) under the Vigoni programme. We wish to thank the anonymous referee for useful remarks that allowed us to improve the presentation of our work.

\section{References}

Bartelmann, M. 1996, A\&A, 313, 697

Bartelmann, M., \& Schneider, P. 2001, Phys. Rep., 340, 291

Bartelmann, M., Steinmetz, M., \& Weiss, A. 1995, A\&A, 297, 1
Bartelmann, M., Huss, A., Colberg, J., Jenkins, A., \& Pearce, F. 1998, A\&A, 330,1

Bartelmann, M., Meneghetti, M., Perrotta, F., Baccigalupi, C., \& Moscardini, L. 2003, A\&A, 409, 449

Bartelmann, M., Doran, M., \& Wetterich, C. 2005,

[arXiv:astro-ph/0507257]

Benson, A. J., Kamionkowski, M., \& Hassani, S. H. 2005, MNRAS, 357, 847

Bond, J., Cole, S., Efstathiou, G., \& Kaiser, N. 1991, ApJ, 379, 440

Brax, P. \& Martin, J. 2000, PRD, 61, 103502

Caldwell, R., Doran, M., Müller, C., Schäfer, G., \& Wetterich, C. 2003, ApJ, 591, L75

Cassano, R. \& Brunetti, G. 2005, MNRAS, 357, 1313

Chandrasekhar, S. 1943, Rev. Mod. Phys., 15, 1

Dalal, N., Hennawi, J., \& Bode, P. 2005, ApJ, 622, 99

Dolag, K., Bartelmann, M., Perrotta, F., et al. 2004, A\&A, 416, 853

Doran, M., Lilley, M., Schwindt, J., \& Wetterich, C. 2001a, ApJ, 559, 501

Doran, M., Schwindt, J., \& Wetterich, C. 2001b, Phys. Rev. D, 64, 123520

Fedeli, C., Meneghetti, M., Bartelmann, M., Dolag, K., \& Moscardini, L. 2006, A\&A, 447, 419

Ferreira, P. \& Joyce, M. 1998, PRD, 58, 023503

Gladders, M., Hoekstra, H., Yee, H., Hall, P., \& Barrientos, L. 2003, ApJ, 593, 48

Goldstein, J., Ade, P., Bock, J., et al. 2003, ApJ, 599, 773

Hasinger, G., Giacconi, R., Gunn, J., et al. 1998, A\&A, 340, L27

Hawkins, E., Maddox, S., Cole, S., et al. 2003, MNRAS, 346, 78

Hennawi, J., Dalal, N., Bode, P., \& Ostriker, J. 2005,

[arXiv: astro-ph/0506171]

Keeton, C. R. 2001, ApJ, 561, 46

Lacey, C. \& Cole, S. 1993, MNRAS, 262, 627

Li, G., Mao, S., Jing, Y., et al. 2005, ApJ, 635, 795L

Manera, M. \& Mota, D. F. 2006, MNRAS, 371, 1373

Maor, I. \& Lahav, O. 2005, J. Cosm. Astropart. Phys., 07, 003

Meneghetti, M., Bolzonella, M., Bartelmann, M., Moscardini, L., \& Tormen, G. 2000, MNRAS, 314, 338

Meneghetti, M., Bartelmann, M., \& Moscardini, L. 2003a, MNRAS, 346, 67

Meneghetti, M., Bartelmann, M., \& Moscardini, L. 2003b, MNRAS, 340, 105

Meneghetti, M., Bartelmann, M., Dolag, K., et al. 2005, A\&A, 442, 413

Mota, D. F. \& van de Bruck, C. 2004, A\&A, 421, 71

Navarro, J., Frenk, C., \& White, S. 1996, ApJ, 462, 563

Navarro, J., Frenk, C., \& White, S. 1997, ApJ, 490, 493

Peebles, P. J. E. \& Ratra, B. 1988, ApJ, 325, L17

Peebles, P. J. E. \& Ratra, B. 2002, Rev. Mod. Phys., 75, 599

Press, W. \& Schechter, P. 1974, ApJ, 187, 425

Randall, S. W., Sarazin, C. L., \& Ricker, P. M. 2002, ApJ, 577, 579

Readhead, A. C. S., Mason, B. S., Contaldi, C. R., et al. 2004, ApJ, 609, 498

Rebolo, R., Battye, R., Carreira, P., et al. 2004, MNRAS, 353, 747

Riess, A., Strolger, L.-G., Tonry, J., et al. 2004, ApJ, 607, 665

Smail, I., Hogg, D. W., Yan, L., \& Cohen, J. G. 1995, ApJ, 449, L105

Somerville, R. S. \& Kolatt, T. S. 1999, MNRAS, 305, 1

Spergel, D., Verde, L., Peiris, H., et al. 2003, ApJS, 148, 175

Spergel, D. N., Bean, R., Dore', O., et al. 2006, [arXiv: astro-ph/0603449]

Tegmark, M., Strauss, M., Blanton, M., et al. 2004, PRD, 69, 103501

Thompson, D., Pozzetti, L., Hasinger, G., et al. 2001, A\&A, 377, 778

Torri, E., Meneghetti, M., Bartelmann, M., et al. 2004, MNRAS, 349, 476

Wambsganss, J., Bode, P., \& Ostriker, J. 2004, ApJ, 606, L93

Wang, P. 2006, ApJ, 640, 18

Wetterich, C. 1988, Nucl. Phys. B, 302, 668

Wetterich, C. 2004, Phys. Lett. B, 594, 17

Zaritsky, D. \& Gonzalez, A. 2003, ApJ, 584, 691

Zeng, D. \& Gao, Y. 2005, [arXiv: astro-ph/0505163] 